\title{
Hyaluronidase: An overview of its properties, applications, and side effects
}

\author{
Hyunwook Jung \\ Gangnam L Plastic Surgery Center, Sejong, Korea
}

Hyaluronidase, an enzyme that breaks down hyaluronic acid, has long been used to increase the absorption of drugs into tissue and to reduce tissue damage in cases of extravasation of a drug. With the increasing popularity of hyaluronic acid filler, hyaluronidase has become an essential drug for the correction of complications and unsatisfactory results after filler injection. For this reason, when performing procedures using hyaluronic acid filler, a sufficient knowledge of hyaluronidase is required. In order for hyaluronidase to dissolve a hyaluronic acid filler, it must interact with its binding sites within the hyaluronic acid. The reaction of a filler to hyaluronidase depends on the hyaluronic acid concentration, the number of crosslinks, and the form of the filler. Hyaluronidase is rapidly degraded and deactivated in the body. Therefore, in order to dissolve a hyaluronic acid filler, a sufficient amount of hyaluronidase must be injected close to the filler. If the filler is placed subcutaneously, injection of hyaluronidase into the filler itself may help, but if the filler is placed within a blood vessel, it is sufficient to inject hyaluronidase in the vicinity of the vessel, instead of into the filler itself. Allergic reactions are a common side effect of hyaluronidase. Most allergic reactions to hyaluronidase are local, but systemic reactions may occur in infrequent cases. Since most allergic responses to hyaluronidase are immediate hypersensitivity reactions, skin tests are recommended before use. However, some patients experience delayed allergic reactions, which skin tests may not predict.

Keywords Hyaluronidase / Hyaluronic acid / Filler
Correspondence: Hyunwook Jung Gangnam L Plastic Surgery Center, SaeromCity Building, 273 Hannuridaero, Sejong 30127, Korea Tel: +82-44-863-1412 Fax: +82-44-864-1413 E-mail: hyunwookj83@gmail.com

This review article was prepared by the Botulinum Toxin-Filler-Thread Academic Association for the Korean Plastic \& Reconstructive Surgeon.

\section{INTRODUCTION}

Hyaluronidase, which is an enzyme that breaks down hyaluronic acid, has been used in medical applications for over 60 years. The US Food and Drug Administration has approved hyaluronidase for the following indications: (1) subcutaneous fluid infusion (hypodermoclysis), (2) as an adjuvant to accelerate the absorption and dispersion of drugs in subcutaneous tissue or to manage extravasation, and (3) as an adjunct to promote the ab- sorption of contrast media in urinary tract angiography (subcutaneous urography) [1]. In addition, it has been approved and used for the purpose of increasing hematoma absorption in Europe [2]. Hyaluronidase has a variety of uses in addition to its approved indications. Its current off-label uses include dissolving hyaluronic acid fillers, treating granulomatous foreign body reactions, and treating skin necrosis associated with filler injections. Although the use of hyaluronidase for these off-label indications has increased significantly, medical practitioners have few op- 
portunities to study hyaluronidase. This article presents the types and characteristics of hyaluronidase, and also introduces research results that will be helpful for using hyaluronidase.

\section{ACTION OF HYALURONIDASE}

Hyaluronic acid is a glycosaminoglycan, a major component of the extracellular matrix, and is also a component of fillers that are frequently used for cosmetic purposes. As shown in Fig. 1, which depicts the structure of hyaluronic acid, D-glucuronic acid and D-N-acetylglucosamine are polymers composed of disaccharides linked by $\beta-1,4$ and $\beta-1,3$ glycosidic bonds. Hyaluronic acid fillers are made by cross-linking hyaluronic acid molecules with a plasticizing agent such as 1,4-butanediol diglycidyl (BDDE) to stabilize and slow decomposition [3].

Hyaluronidase is an endoglycosidase that breaks down hyaluronic acid into monosaccharides by cleaving its glycosidic bonds; additionally, to some extent, it also breaks down other acid mucopolysaccharides in the connective tissue [4].

\section{TYYPES OF HYALURONIDASE}

Human hyaluronidase is present both in organs (testis, spleen, skin, eyes, liver, kidneys, uterus, and placenta) and in body fluids (tears, blood, and semen) [5]. There are six known types (hyaluronidase 1-4, PH-20, and HYALP1). Hyaluronidase 1, which is encoded by the HYAL1 gene, is present in major organs such as the liver, kidney, spleen, and heart, as well as in serum and urine. It acts as a major hyaluronidase in plasma and is activated at an acidic $\mathrm{pH}$. Hyaluronidase 2 exerts weaker enzymatic activ- ity than hyaluronidase 1 and only breaks down high-molecularweight hyaluronic acid. Hyaluronidase 3 is found only in the testis and bone marrow, and its role is unknown. Testicular $\mathrm{PH}$ 20 hyaluronidase is found on the surface of human sperm and inner acrosomal membrane and serves to degrade hyaluronic acid in the ovum during fertilization [6].

Meyer classified hyaluronidases into three categories according to its mechanism of action. First, mammalian hyaluronidases are endo- $\beta$-N-acetylhexosaminidases that break down $\beta-1,4$ glycosidic linkages to form tetrasaccharides. Second, leech/hookworm hyaluronidases are endo- $\beta$-D-glucuronidases that break down $\beta-1,3$ glycosidic bonds to form pentasaccharides and hexasaccharides. Finally, microbial hyaluronidases are classified as hyaluronate lyases. Unlike other hyaluronidases, they do not catalyze hydrolysis reactions; instead, they produce unsaturated disaccharides through a $\beta$-elimination reaction at $\beta-1,4$ glycosidic linkages (Fig. 1) [2].

Hyaluronidases can also be classified into two types according to the $\mathrm{pH}$ at which they are most active. Acid-active hyaluronidases are activated at a pH of 3 to 4 . Neutral-active hyaluronidases - which includes the hyaluronidase enzymes found in snake and bee venom-are activated at a $\mathrm{pH}$ of 5 to 8 [2].

In the past, medical hyaluronidase was extracted from bovine or sheep testicles and used without purification. However, the mammalian hyaluronidase obtained in this way was low in purity and contained components that could cause an immune response. Subsequently, purification of mammalian hyaluronidase was implemented as a processing step, and microbial hyaluronidase obtained from Streptococcus agalactiae bacteria was also used to reduce side effects.

\section{Fig. 1. Structure of hyaluronic acid}

D-Glucuronic acid and D-N-acetylglucosamine are linked by $\beta-1,3$ bonds (blue) to form a disaccharide. Multiple disaccharides are linked by $\beta-1,4$ bonds (red) to form hyaluronic acid. Mammalian and microbial hyaluronidases cleave $\beta-1,4$ bonds (red), and leech/hookworm hyaluronidases degrade $\beta-1,3$ bonds (blue).

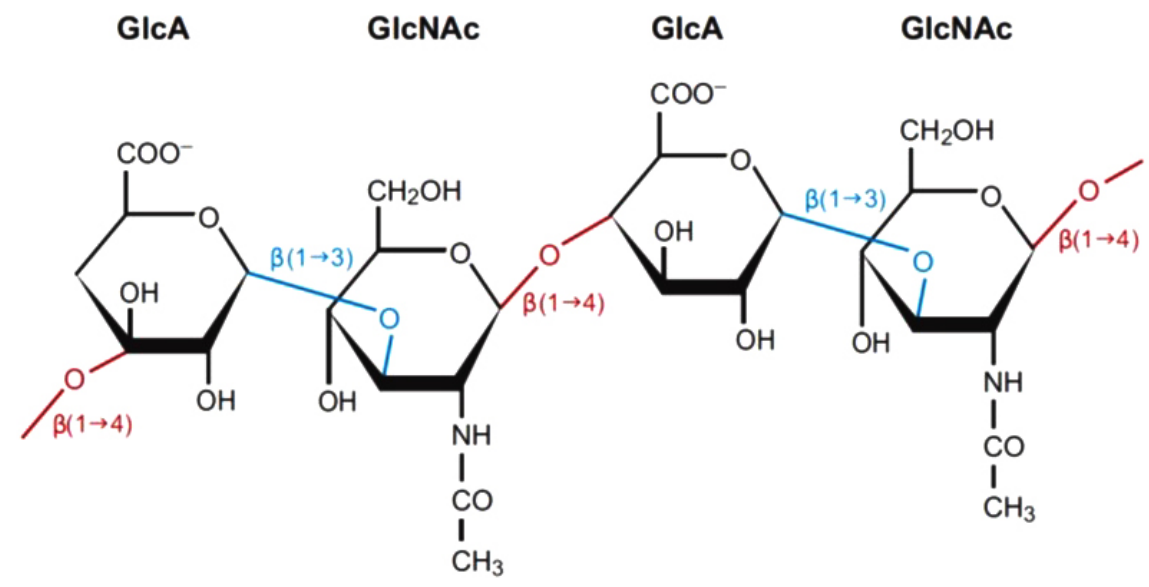




\section{HYALURONIDASE IN THE BODY}

When hyaluronidase is injected into the body, its activity gradually decreases over time as a result of dilution, diffusion, and deactivation [7]. Deactivation is caused by anti-hyaluronidase activity, which proceeds at different rates in subcutaneous tissue and the plasma. In an experiment using rodents, the half-life of hyaluronidase in subcutaneous tissue was less than 30 minutes $[8,9]$, and its activity was partially maintained until 1 hour depending on the experiment [10]. In plasma, the half-life was 2 to 3 minutes when hyaluronidase was injected intravenously in humans, and even repeated injections did not result in a sustained elevation of serum levels of hyaluronidase $[2,5,8]$. The reasons for the short half-life of hyaluronidase in human plasma are the presence of numerous hyaluronidase inhibitors in the plasma and the metabolism of hyaluronidase in the kidneys and liver [2].

Hyaluronidase in the body is affected by various drugs. Hyaluronidase antagonists include anti-inflammatory agents (e.g., indomethacin, dexamethasone, and salicylates), numerous plantbased compounds (e.g., flavonoids and antioxidants), antihistamines, mast cell stabilizers, heparin, vitamin C, dicumarene, and radiographic contrast media $[2,5]$.

Levels of hyaluronidase inhibitors may increase depending on an individual's physical condition. In acute-phase responses such as burns, septicemia, and shock, hyaluronidase inhibitor levels increase to prevent circulatory collapse by reducing the turnover rate of hyaluronic acid [11].

\section{HYALURONIC ACIID FILLERS ANID HYALURONIDASE}

In order for hyaluronidase to dissolve a hyaluronic acid filler, it must be able to access the intramolecular bonds within hyaluronic acid. The factors that interfere with access include the number of crosslinks between hyaluronic acid molecules and the concentration of hyaluronic acid. The more cross-linking, the more difficult it is for hyaluronidase to access its binding sites inside the hyaluronic acid filler. For this reason, fillers with extensive cross-linking require a long time to dissolve with hyaluronidase [12]. In addition, the higher the concentration of hyaluronic acid, the slower it will be dissolved by hyaluronidase [13]. Monophasic fillers are less soluble in hyaluronidase because they are less exposed to hyaluronidase than polyphasic fillers [3].

As the side effects of fillers on blood vessels are known, many attempts have been made to effectively dissolve fillers inside blood vessels. DeLorenzi [3] concluded in a 2014 paper that hyaluronidase could be injected subcutaneously without the need for intravascular injections to treat filler-induced vascular embolism. In an animal experiment, Wang et al. [14] also found that subcutaneous injections of hyaluronidase were more effective than intravascular injections for preventing skin necrosis caused by hyaluronic acid filler embolism. The amount of hyaluronidase is important when injecting hyaluronidase in the vicinity of a blood vessel to dissolve filler inside the vessel. Lee et al. [15] reported that it was effective to inject 30-50 IU or more in one place in an animal test in 2020, and recommended injecting $100 \mathrm{IU}$ or more at each location for a clear effect.

\section{SIDE EFFECTS OF HYALURONIDASE}

Local injections of hyaluronidase can cause side effects such as local pruritus and allergic reactions. The incidence of allergic reactions is reported to be $0.05 \%$ to $0.69 \%$, and urticaria and angioedema have also been reported to occur at a low frequency (less than $0.1 \%)[2,16]$. Allergic reactions are more likely to occur when the hyaluronidase dose is more than 100,000 IU through an intravenous injection, and the occurrence of allergic complications rises to $31.3 \%$ if the dose increases to $200,000 \mathrm{IU}$ [2].

Most allergic reactions of hyaluronidase are immediate hypersensitivity reactions (type I, immunoglobulin E-mediated), but delayed hypersensitivity reactions (type IV, T-cell-mediated) may also occur [17]. Immediate hypersensitivity reactions caused by hyaluronidase manifest as erythematous edema after 1 to 2 hours, and there is no response to antibiotic treatment. In such cases, systemic steroids, antihistamines, and steroid cream application are helpful [17]. Delayed hypersensitivity reactions caused by hyaluronidase can occur even after 24 hours, and in such cases, a skin test will not produce a positive reaction within 20 minutes, resulting in a negative diagnosis [18].

The skin test is performed with 3 IU of hyaluronidase; although is recommended to perform a skin test before using hyaluronidase, doing so can often be difficult in general clinics [17]. There is usually no link between the patient's history of allergies and the response to hyaluronidase. However, depending on the origin of hyaluronidase, injection of hyaluronidase should be avoided because cross-reactions may occur in patients who are allergic to bovine collagen and bee stings [1]

\section{CONCLUSIONS}

Hyaluronidase use has become more diverse and widespread in clinical practice. In particular, it is used to address patients' dissatisfaction after hyaluronic acid filler treatment and to treat side effects. As its use becomes more common, it is increasingly im- 
portant for clinicians to have a sufficient knowledge of hyaluronidase. Hyaluronidase can serve as an appropriate treatment in a variety of situations if it is used with a thorough understanding of its mechanism of action, metabolism, and side effects.

\section{NOTES}

\section{Conflict of interest}

No potential conflict of interest relevant to this article was reported.

\section{ORCID}

HyunwookJung https://orcid.org/0000-0002-1186-7510

\section{REFERENCES}

1. Bailey SH, Fagien S, Rohrich RJ. Changing role of hyaluronidase in plastic surgery. Plast Reconstr Surg 2014;133:127e$132 \mathrm{e}$.

2. Cavallini M, Gazzola R, Metalla M, et al. The role of hyaluronidase in the treatment of complications from hyaluronic acid dermal fillers. Aesthet Surg J 2013;33:1167-74.

3. DeLorenzi C. Transarterial degradation of hyaluronic acid filler by hyaluronidase. Dermatol Surg 2014;40:832-41.

4. Rzany B, Becker-Wegerich P, Bachmann F, et al. Hyaluronidase in the correction of hyaluronic acid-based fillers: a review and a recommendation for use. J Cosmet Dermatol 2009; 8:317-23.

5. Menzel EJ, Farr C. Hyaluronidase and its substrate hyaluronan: biochemistry, biological activities and therapeutic uses. Cancer Lett 1998;131:3-11.

6. Jiang D, Liang J, Noble PW. Hyaluronan in tissue injury and repair. Annu Rev Cell Dev Biol 2007;23:435-61.

7. DeLorenzi C. New high dose pulsed hyaluronidase protocol for hyaluronic acid filler vascular adverse events. Aesthet Surg J 2017;37:814-25.

8. Zhu GZ, Sun ZS, Liao WX, et al. Efficacy of retrobulbar hy- aluronidase injection for vision loss resulting from hyaluronic acid filler embolization. Aesthet Surg J 2017;38:12-22.

9. Wasserman RL. Overview of recombinant human hyaluronidase-facilitated subcutaneous infusion of $\operatorname{IgG}$ in primary immunodeficiencies. Immunotherapy 2014;6:553-67.

10. Kim HJ, Kwon SB, Whang KU, et al. The duration of hyaluronidase and optimal timing of hyaluronic acid (HA) filler reinjection after hyaluronidase injection. J Cosmet Laser Ther 2018;20:52-7.

11. Mio K, Carrette O, Maibach HI, et al. Evidence that the serum inhibitor of hyaluronidase may be a member of the inter-alpha-inhibitor family. J Biol Chem 2000;275:32413-21.

12. Rao V, Chi S, Woodward J. Reversing facial fillers: interactions between hyaluronidase and commercially available hyaluronic-acid based fillers. J Drugs Dermatol 2014;13:10536.

13. Jones D, Tezel A, Borrell M. In vitro resistance to degradation of hyaluronic acid dermal fillers by ovine testicular hyaluronidase. Dermatol Surg 2010;36:804-9.

14. Wang M, Li W, Zhang Y, et al. Comparison of intra-arterial and subcutaneous testicular hyaluronidase injection treatments and the vascular complications of hyaluronic acid filler. Dermatol Surg 2017;43:246-54.

15. Lee W, Oh W, Oh SM, et al. Comparative effectiveness of different interventions of perivascular hyaluronidase. Plast Reconstr Surg 2020;145:957-64.

16. Dunn AL, Heavner JE, Racz G, et al. Hyaluronidase: a review of approved formulations, indications and off-label use in chronic pain management. Expert Opin Biol Ther 2010; 10:127-31.

17. Kim MS, Youn S, Na CH, et al. Allergic reaction to hyaluronidase use after hyaluronic acid filler injection. J Cosmet Laser Ther 2015;17:283-5.

18. Feighery C, McCoy EP, Johnston PB, et al. Delayed hypersensitivity to hyaluronidase (Hyalase) used during cataract surgery. Contact Dermatitis 2007;57:343. 